\title{
Gravitational instability and clustering in a disk of planetesimals
}

\author{
P. Tanga ${ }^{1, \star}$, S. J. Weidenschilling ${ }^{2}$, P. Michel ${ }^{1}$, and D. C. Richardson ${ }^{3}$ \\ ${ }^{1}$ Laboratoire Cassiopée CNRS/UMR6202, Observatoire de la Côte d'Azur, BP 4229, 06304 Nice Cedex 04, France \\ e-mail: tanga@obs-nice.fr \\ 2 Planetary Science Institute, 1700 East Fort Lowell Road, Suite 106, Tucson, AZ 85719-2395, USA \\ 3 Dept. of Astronomy, Univ. of Maryland, College Park, MD 20742-2421, USA
}

Received 15 April 2004 / Accepted 5 August 2004

\begin{abstract}
For a long time, gravitational instability in the disk of planetesimals has been suspected to be the main engine responsible for the beginning of dust growth, its advantage being that it provides for rapid growth. Its real importance in planetary formation is still debated, mainly because the potential presence of turbulence can prevent the settling of particles into a gravitationally unstable layer. However, several mechanisms could yield strongly inhomogeneous distributions of solids in the disk: radial drift, trapping in vortices, perturbations by other massive bodies, etc. In this paper we present a numerical study of a gravitationally unstable layer. This allows us to go beyond the classical analytical study of linear perturbations, exploring a highly non-linear regime. A hierarchical growth of structure in the presence of dissipation (gas drag) can yield large, virialized clusters of planetesimals, the first time such clusters have been observed in the context of planetesimal disks.
\end{abstract}

Key words. stars: planetary systems: protoplanetary disks - gravitation - planets and satellites: formation

\section{Introduction}

The formation of planetesimals from dust in the solar nebula, and their growth to form bodies of planetary size, is a complex process. In the earliest stages of this process, dust-gas interactions, collective effects, and timescales are topics of intense research. Controversy persists as to whether the first solid bodies grew solely by physical collisions and sticking, by gravitational instability in a layer of dust (or larger particles), or by some combination of these processes.

The first detailed qualitative description of planetesimal formation was due to K. E. Edgeworth, who is better known for having postulated the existence of a huge reservoir of cometary bodies beyond Neptune's orbit (the so-called EdgeworthKuiper Belt). He inferred that the settling of particles toward the central plane of a disk-shaped nebula would produce a layer with density much greater than that of the gas, with sufficient density to become unstable due to self-gravity (Edgeworth 1949). In order for this instability to occur, the mutual attraction of the particles had to overcome both the tidal pull of the Sun and the "internal heat" associated with their velocity dispersion. The resultant clusters of particles could then collapse into solid bodies, which would collide and coalesce into larger and larger bodies, eventually producing the planets.

The first mathematical analyses of this process were produced by Safronov (1969), and independently by

* P.T. acknowledges the support of the Poincare fellowship of the Observatoire de la Côte d'Azur and of the Programme National de Planétologie.
Goldreich \& Ward (1973). They performed linear stability analyses of a particle layer with Keplerian rotation. They showed that the particle velocity dispersion stabilized density perturbations of small spatial scale, while rotation acted to stabilize large ones. That is to say, there was a preferred scale for gravitational instabilities, which would tend to produce condensations with a characteristic size. These analyses assumed that there was no obstacle to settling of particles, so that the particle layer could attain the critical density. However, the presence of any kind of turbulence in the gas, as is generally accepted as an unavoidable source of viscosity in accretion disks, could inhibit sedimentation of small particles. Even in a purely laminar nebula, settling of particles into a dense layer could be inhibited. The nebular gas necessarily has a slightly sub-Keplerian velocity due to the existence of a radial pressure gradient. If a particle layer in the midplane attains a density greater than that of the gas, it lacks pressure support and tends toward more nearly Keplerian rotation. The resulting shear generates turbulence that may prevent further settling, so that the critical density for gravitational instability is not reached (Weidenschilling 1980). However, the effectiveness of this process is currently under debate (Youdin \& Shu 2002; Weidenschilling 2003). If the dust/gas ratio can be enhanced several times over solar abundance, by global transport (Youdin \& Shu 2002) or local shear instabilities (Goodman \& Pindor 2000), it may be possible to attain the critical density. It may be achieved more simply if some degree of particle coagulation occurs, as decimeter-sized or larger bodies will decouple form shear-induced turbulence. 
Attainment of the critical density allows gravitational instability, but does not guarantee it. A realistic system of particles would contain bodies with a range of sizes; as the gas drag produces size-dependent radial velocities, there will be some velocity dispersion. This can inhibit instability, until the the mean size becomes larger than a threshold size, estimated by Weidenschilling (1995) to be of the order of $10 \mathrm{~m}$. However, the outcome of gravitational instability in that case may be significantly different from that experienced in a layer of small particles. Goldreich \& Ward (1973) assumed cm-sized particles, which would be subject to damping by collisions and gas drag, and set the velocity dispersion to zero, allowing instabilities on all length scales below the limit set by rotation. In contrast, 10-m bodies are only weakly damped, and may retain a significant velocity dispersion that favors instabilities at a single scale length (Weidenschilling 1995). Numerical simulations of particle coagulation in the outer solar system, where cometary nuclei presumably accreted, showed that gravitational instability could have occurred in such an environment (Weidenschilling 1997; henceforth W97). At 30 AU from the Sun, the size of the unstable regions would be $\sim 0.016 \mathrm{AU}$ for a low-mass solar nebula, containing $\sim 10^{22} \mathrm{~g}$ of condensed matter. Weidenschilling speculated that clusters of decameter-sized bodies on this scale would not collapse to solid bodies with diameters $\sim 100 \mathrm{~km}$; rather, he suggested that due to the lack of damping such condensations would be transient. However, the detailed evolution of such clusters, their duration, and their possible influence on the growth of planetesimals have not been explored by numerical simulations. In general, two elements play against gravitational collapse: the overall velocity dispersion, if not damped by collisions or gas drag, and the angular momentum of a gravitationally bound cluster due to the Keplerian rotation of the particle layer. If both of these quantities are too large, the first can prevent instability on small scales, while the second can prevent collapse beyond a certain upper-limit scale.

As detailed in Sect. 2, all these factors are taken into account in the linear perturbation analysis of the stability of a nearly homogeneous disk. However, the subsequent evolution can be highly non-linear and its study requires the use of numerical simulations.

Given the long-lasting interest in the general problem of gravitational instability and its importance for our understanding of the planetesimal growth process, both in our solar system and elsewhere, we present in this work the numerical study of the evolution of a gravitationally unstable layer of planetesimals in orbit around the Sun. Simulating the process allows us to explore the collective evolution, going beyond the linear approximation usually employed to describe the onset of gravitational instability, and to study the dynamics of planetesimal clusters. A fully consistent simulation of the W97 scenario is, however, well beyond the current computational limits. In fact it is simply not possible to simulate a sufficiently large disk patch at $30 \mathrm{AU}$ with a number of particles, distributed in a range of sizes, large enough to reproduce the evolution and accretion of a planetesimal swarm starting from $1 \mathrm{~cm}$ or $1 \mathrm{~m}$ bodies.

We use the $N$-body hierarchical tree code pkdgrav, (Richardson et al. 2000) which is able to treat physical collision between particles in a consistent way, simulating their coalescence into larger bodies. Given the large numbers of interactions and particles involved, all the runs presented here required the use of high-performance parallel computers ${ }^{1}$. The unstable regime was explored both in a gas-free environment and in the presence of a Keplerian non-turbulent gas disk. We note that the results obtained here can also be helpful for understanding the process of gravitational collapse in other scenarios where gravitational instability could play a role, such as those invoking particle concentration in the core of large-scale anticyclonic vortices (Barge et al. 1995; Tanga et al. 1996).

In the following section we will address the numerical method employed, together with boundary conditions. Later (Sect. 3) we illustrate the evolution of a layer of single-sized large planetesimals, considering either energy-conserving (perfectly elastic) mutual collisions or inelastic bouncing. In Sect. 4 we show the effect of enhancing dissipation by including the gas drag exerted by a laminar, Keplerian flow.

\section{Numerical framework}

The pkdgrav parallel $N$-body code allows in principle for treatment of a large number $(N)$ of particles, and numerical experiments have been successfully performed with $N$ ranging from $10^{5}$ up to $10^{6}$ (see for example Richardson et al. 2000; Tanga et al. 2002). The main advantage provided by this code for our purpose is the possibility to assign a physical size to particles (considered to be spherical) and to efficiently detect mutual collisions. The collisional outcome can be treated in different ways. In the following we will show results obtained either by imposing perfectly elastic bouncing between the colliders, or by making the colliders merge into a single body of mass equal to the sum of their masses, with the same internal density. Considering the colliding bodies as an isolated system, the first case implies energy conservation in the reference frame referred to the center of mass. In the second case, dissipation occurs. Accordingly, we will refer to the two families of simulations with the acronyms "EC" (for "energy conserving" collisions) and "CM" (for "collisional merging").

All the simulations presented in the following have been performed in the approximation introduced by Wisdom \& Tremaine (1988), that is by assuming that a dense ring of planetesimals at distance $a$ from the central body can be divided into local boxes (or "patches") of size $L$, with $L \ll a$. In a coordinate frame centered on the box, with the $x$-axis pointing away from the Sun, the $y$-axis being tangential to the direction of motion, and the $z$-axis perpendicular to the orbital plane, the equation of motion for one particle can be linearized:

$$
\begin{aligned}
& \ddot{x}=F_{x}+3 \Omega^{2} x+2 \Omega \dot{y}, \\
& \ddot{y}=F_{y}-2 \Omega \dot{x}, \\
& \ddot{z}=F_{z}-\Omega^{2} z,
\end{aligned}
$$

in which $\Omega$ is the angular frequency at distance $a$ from the Sun, and $F$ represents the gravitational force (per unit mass) due to the other particles.

\footnotetext{
In particular, we wish to acknowledge the use of SIVAM-II at the Observatoire de la Côte d'Azur, of the PC cluster installed in the same institute by ALINEOS, and of the IDRIS computing facilities.
} 
The box in which the simulation is performed is considered to be periodic in the $(x, y)$ plane. In order to reduce boundary effects, the gravitational contribution coming from three orders of ghost patches, each containing a copy of the particles in the main domain, is considered. The ghost patches are nested around the central one; those having a distance from the Sun different from $a$ have a velocity tangential to the orbit consistent with the Keplerian shear.

It must be noted that the collective particle behavior can be associated to structures (such as clusters, waves, etc.) whose typical scale length must be much smaller than $L$ to avoid boundary effects. However, given the limited resolution available in terms of $N$, it may happen that adequate sampling of all interesting scales present in the disk is not possible. In other words, while the largest scales are always limited by the size of $L$, the smallest possible scales that can be represented in the simulation have a size corresponding to several times the average particle diameter.

The spatial scales that we are interested in can be estimated from the linear theory of gravitational instability in a rotating disk of planetesimals (in the following, we use the notation of Ward 1976). The dispersion relation that is obtained, in the case of a thin disk locally rotating at frequency $\Omega$, for a slightly overdense domain of size $\lambda$, can be written as:

$F(\lambda)=2 \pi^{2} c^{2}-4 \pi^{2} G \Sigma \lambda+\lambda^{2} \Omega^{2}<0$,

in which $c$ represents the velocity dispersion and $\Sigma$ the local surface density of solids. An unstable solution exists when $F(\lambda)<0$, i.e., for $c<c_{\text {crit }}=\pi G \Sigma / \Omega$. The widest range of unstable wavelengths is obtained when $c=0$; in that case, for $\lambda<2 \lambda_{\text {crit }}$ instability is possible. $\lambda_{\text {crit }}$ is the value of the most unstable wavelength (minimum of the dispersion relation) and is given by:

$\lambda_{\text {crit }}=\frac{2 \pi^{2} G \Sigma}{\Omega^{2}}$

As will be shown, the overdense regions grow by hierarchical merging into larger and larger structures. However, before their sizes become comparable to $L$, the increase in velocity dispersion stabilizes the dust layer, so that these structures dissipate.

\section{Unstable structure growth in a "cold" disk}

We simulate here a patch of the disk at an average distance from the Sun of $a_{0}=30$ AU. Since the observed structures develop on scales $\ll \lambda_{\text {crit }}$, a box size comparable to $\lambda_{\text {crit }}$ does not appear to be a serious limitation. In the following, we choose a patch size $L=0.04 \mathrm{AU}$, i.e., $\sim 70 \%$ larger than the critical wavelength, $\lambda_{\text {crit }} \sim 0.024 \mathrm{AU}$. We checked one case with $L=$ $7 \lambda_{\text {crit }}$, but no qualitative difference was noted in the evolution of structures. We are thus confident that the results presented here are not seriously affected by boundary effects.

The imposed initial conditions are derived from the comet formation model in W97 after $t \sim 10^{5} \mathrm{yr}$ of model evolution. At that stage, a theoretically unstable layer is formed. This choice is just a reasonable starting point for investigating the dynamics of planetesimals in the comet-forming region even though, as we will see, the limitations in computing power prevent a direct comparison to the W97 scenario.

The conditions for the onset of the instability require a density above a certain critical value, so that self-gravity can overcome the solar tides that otherwise have the tendency to shear away all structures. This critical density is usually written as:

$\rho_{\text {crit }}=\frac{3 M}{2 \pi a^{3}}=\frac{3 \Omega^{2}}{2 \pi G}$.

The initial vertical dispersion $h$ chosen in our simulations corresponds to a volume density $\rho \sim 16 \times \rho_{\text {crit }}$, consistent with W97. The initial positions of the particles are generated by assigning random positions inside a $L \times L \times h$ box, avoiding particle overlaps. The components of the velocity dispersion on the disk plane, that we indicate by $c_{x}$ and $c_{y}$, are initially set equal to zero. As a consequence, the particles start on ideal, circular Keplerian orbits. Concerning $c_{z}$, its value is imposed to be consistent with the vertical dispersion.

The surface density is $\Sigma=0.41 \mathrm{~g} \mathrm{~cm}^{-2}$. W97 states that "about two-thirds" of the total solids take part in the instability. However, we did not reduce the average surface density accordingly, preferring to let the dynamics of the layer determine the evolution of the entire mass.

A good compromise between the simulation speed, the number of particles and the time step was found for $N=$ $4 \times 10^{4}$, with $1.6 \times 10^{5}$ steps per revolution. This allows for computation of the dynamical evolution over a few orbits in about $100 \mathrm{~h}$ of CPU on a cluster of four SP4 processors.

In order to reproduce the surface density given above, with the material bulk density of particles set to $1 \mathrm{~g} \mathrm{~cm}^{-3}$, the particle radius must be taken equal to $R=10 \mathrm{~km}$. This size is about 100 times the particle size that is built at $t \sim 10^{5} \mathrm{yr}$ in the model of W97. The consequence is a stronger gravitational stirring: we can thus expect that, in the absence of any dissipation, the vertical dispersion of the planetesimal disk will rapidly increase beyond the initially imposed value.

Because of this large size, it is clear that we will not be able to obtain a realistic simulation of the comet-forming region. A direct comparison with the scenario of W97 is thus not straightforward. Nevertheless, we can infer some interesting properties of the collapsing layer, and shed some light on previously ignored behaviors.

In this case, the EC and the CM simulations have several features in common. According to (3), the small wavelengths emerge first (Fig. 1). The growth of structures at larger scales is associated with the gradual coalescence of larger and larger structures, similar to what happens during the process of hierarchical growth already observed in numerical simulations of cosmological structures. The main difference with cosmology, here, is the deformation of clusters due to shear, translating into the elongated structures that in the context of planetary rings are known as "wakes" (see e.g., Richardson 1994). Later, the structures dissipate at all scales. This sequence of events is evident in Fig. 1, which shows snapshots from the CM case. The instability, here, is not sufficient to create conditions for an efficient local collapse, and the size distribution of the CM simulation (Fig. 2) shows that only a small fraction of the population has moderately grown in size. 

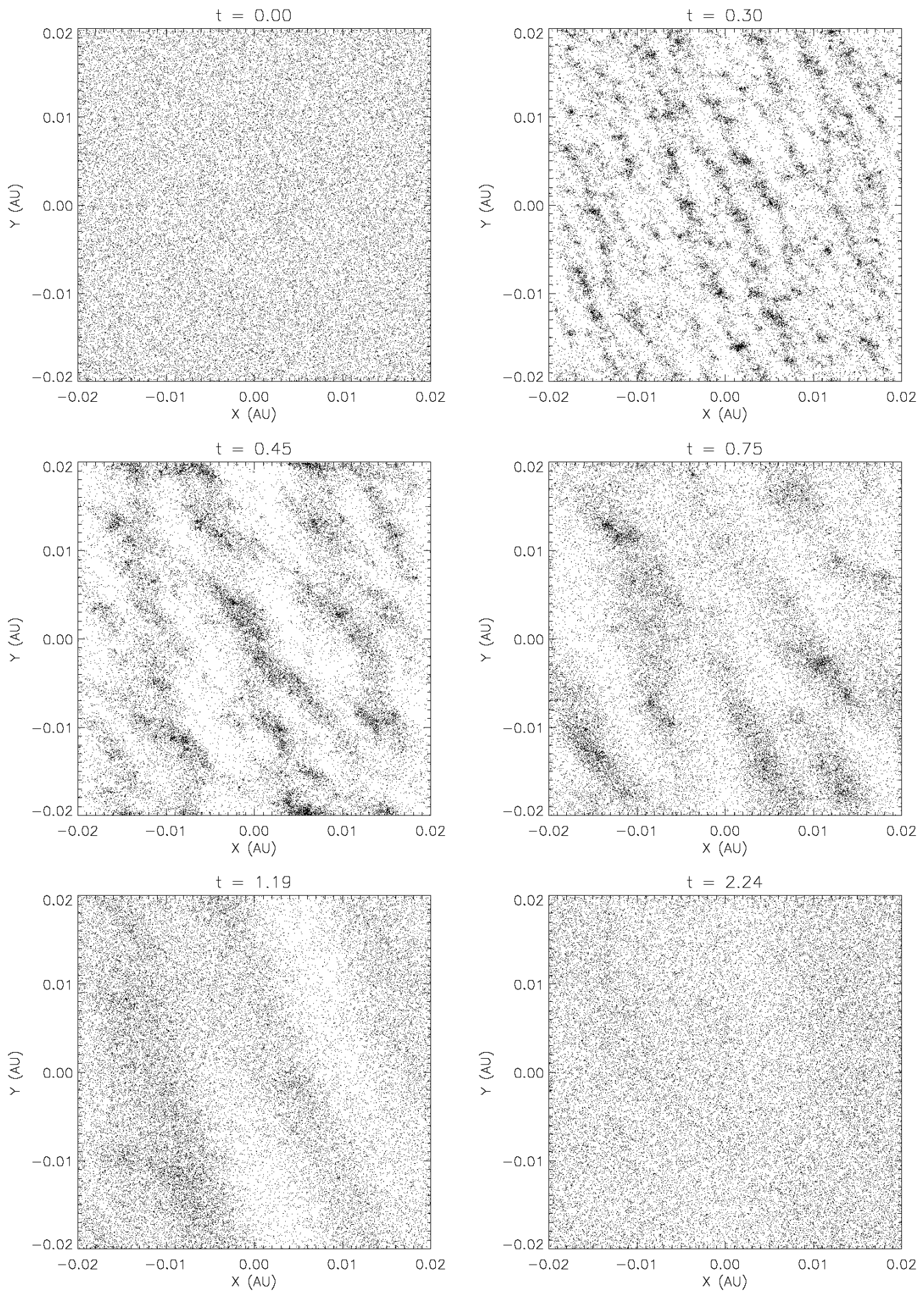

Fig. 1. Particles positions as projected on the $(x, y)$ plane in the CM simulation. The first panel represents initial conditions. Time is expressed in orbital periods (at $30 \mathrm{AU}$, about 164 years).

In order to get some quantitative estimate of the local density enhancement, we computed the surface density $\Sigma(n, m)$ on a $N \times M$ grid ( $N$ being the dimension along $x$ ). Figure 3 (upper panel) shows the time evolution of the maximum density found in the domain, using $N=M=32$. A peak corresponding to a ten-fold enhancement can be identified at $t \sim 0.42$ orbit. As expected from visual inspection, at later times the distribution slowly recovers its initial homogeneity. This can be seen also in a histogram of the density at different times (Fig. 3, lower panel), which shows that the rapid initial clustering creates strongly overdense regions and a large population of nearly empty areas (dashed line). Later, the peak of the distribution gradually returns toward the average value and the tail at large densities is lost.

An estimate of the wavelengths of the structures at given times can be obtained by computing the power spectrum of the density distribution. In principle, this could be derived from the 2D Fourier transform (FT) of the surface density field $\Sigma(n, m)$ 


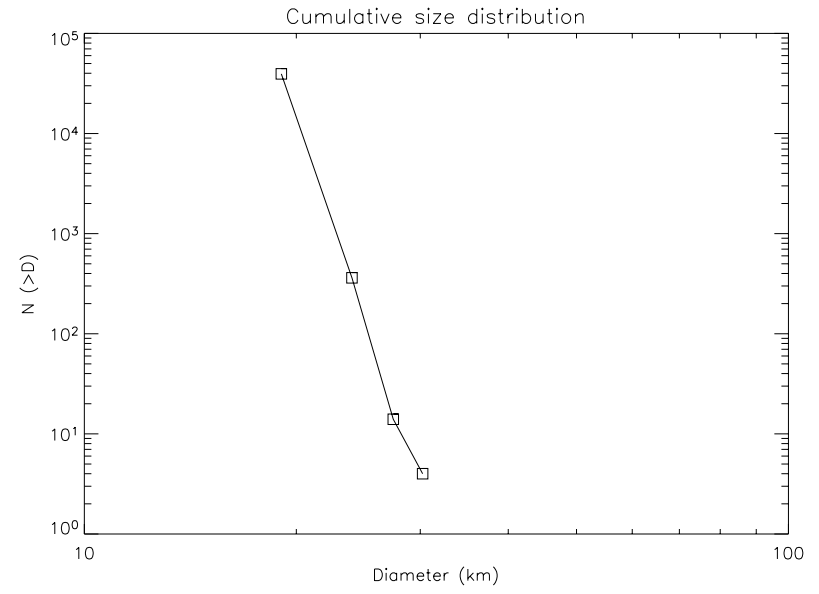

Fig. 2. Cumulative size distribution for the CM simulation at the end of the run ( $t=2.24$ orbits).

in the disk patch. However, since the structures are stretched along the $y$ direction by the Keplerian shear, a 1D FT along $x$ is easier to interpret and compute. The power spectrum was thus computed as the average of the FT of the $M$ rows:

$\mathcal{P}(k)=\frac{1}{M} \sum_{i=1}^{M}\|\mathcal{F}(\Sigma(n, i))\|$,

where $\mathcal{F}$ represents the Fourier transform. In this case we chose $N=128, M=40$ and we applied an FFT algorithm.

The results thus obtained are summarized in Fig. 4. As expected the power spectrum of the initial conditions corresponds to white noise. However, the evolution shows quite well the energy accumulating in larger and larger wavelengths, as shown by the displacement of the maximum of the power spectrum. The last (upper) curve shows that when large structures are present, a flat spectrum is recovered at high frequencies, indicating the disappearance of small scale structures.

The time evolution of the wavelength corresponding to the maximum of the power spectrum is shown in Fig. 5. The noise associated to the limited resolution does not allow for precise conclusions to be drawn on the trend. Qualitatively, all we can observe is that it is not possible to detect significant departures from linear growth.

As shown also in Fig. 6, the system evolves toward an equilibrium state at which velocity dispersions have stabilized all the wavelengths. Here, the velocity dispersion $c$ used to compute the dispersion relation (Eq. (2)) is the quadratic average of the three components $c_{x, y, z}$. Each component is obtained by the mass-weighted dispersion, i.e., by computing:

$c=\sqrt{\frac{\sum_{i} m_{i}\left(v_{i}-\langle v\rangle\right)^{2}}{\sum m_{i}}}$,

in which $i$ is the index referred to particles, and sums are computed over the total particle number. The initial ordered motion in the dynamically "cold" disk has been transformed in a few orbits into a warmer swarm of particles, as a result of gravitational stirring. At the end of the run, it is clear that the instability is no longer possible; being favored by the entropy increase, the current state is stable.
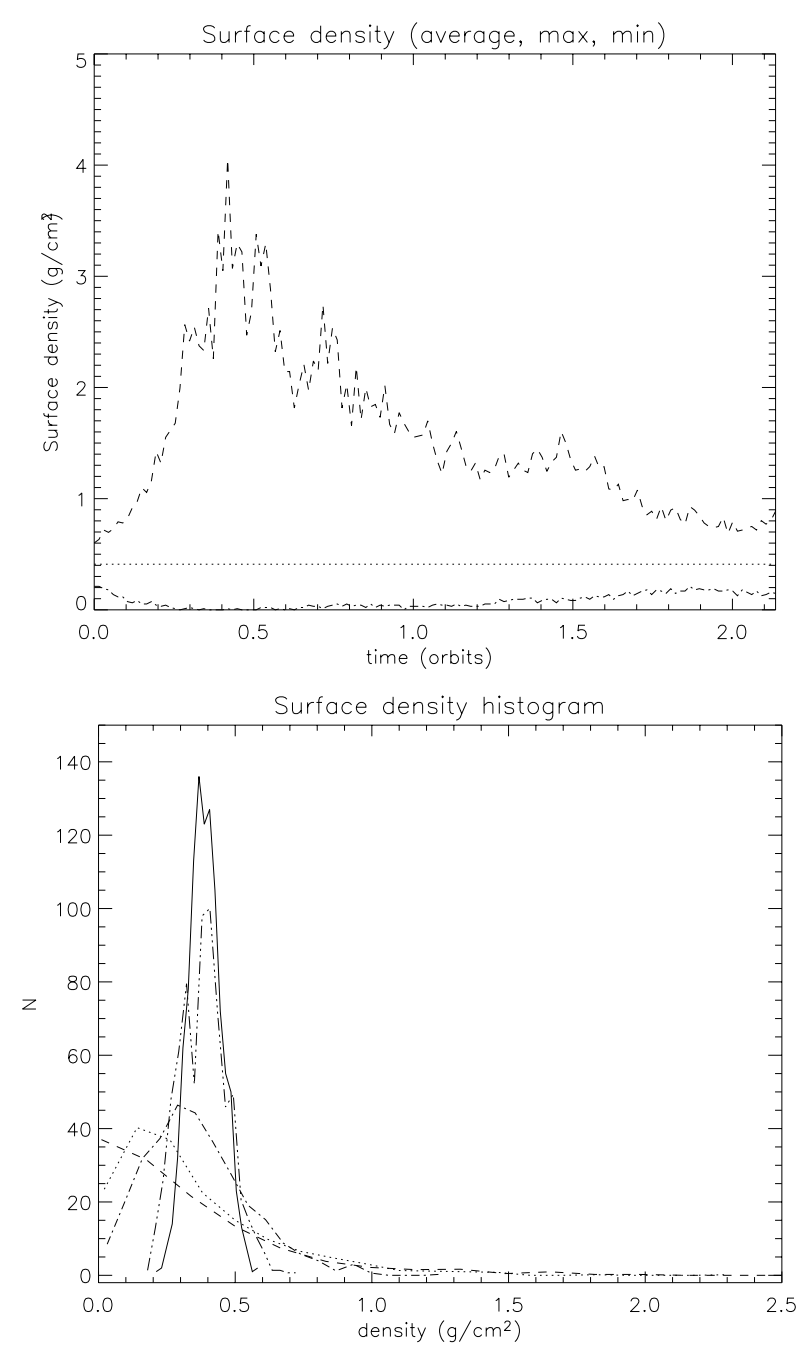

Fig. 3. The two panels show the time evolution of the local surface density, computed on a regular grid of $32 \times 32$ boxes, in the simulation of Fig. 1. The upper panel shows the evolution in time of the maximum and the minimum value; the horizontal dotted line corresponds to the average. The lower panel shows the histogram of the density values at different times. The continuous line refers to the initial conditions $(t=0)$. The dashed line (with maximum at 0 ) refers to $t=0.30$ orbit, the dotted to $t=0.45$ orbit, the dash-dotted to $t=0.74$ orbit. The remaining line, at the end of the evolution ( $t=2.25$ orbits) approaches again the original Gaussian distribution, centered on the average value.

Until now we have not discussed the dissipative effect of inelastic collisions, resulting in particle growth and mergers. However, the EC simulation does not show any difference in comparison to the analysis shown here. Collisions and very close encounters thus seem not relevant for the evolution in the short timescale corresponding to the lifetime of the observed density waves. This can also be seen by considering the timescale due to damping by mutual collisions. Following Ward (1976), this timescale can be written as:

$\tau_{\text {damp }} \sim \frac{4}{3} \frac{\rho_{p} R}{\Sigma} \frac{1}{\beta \Omega}$

in which $\beta$ represents the fraction of energy dissipated at each collision. In our case, having $\beta=1, \tau_{\text {damp }} \sim 3 \times 10^{3}$ orbits, 


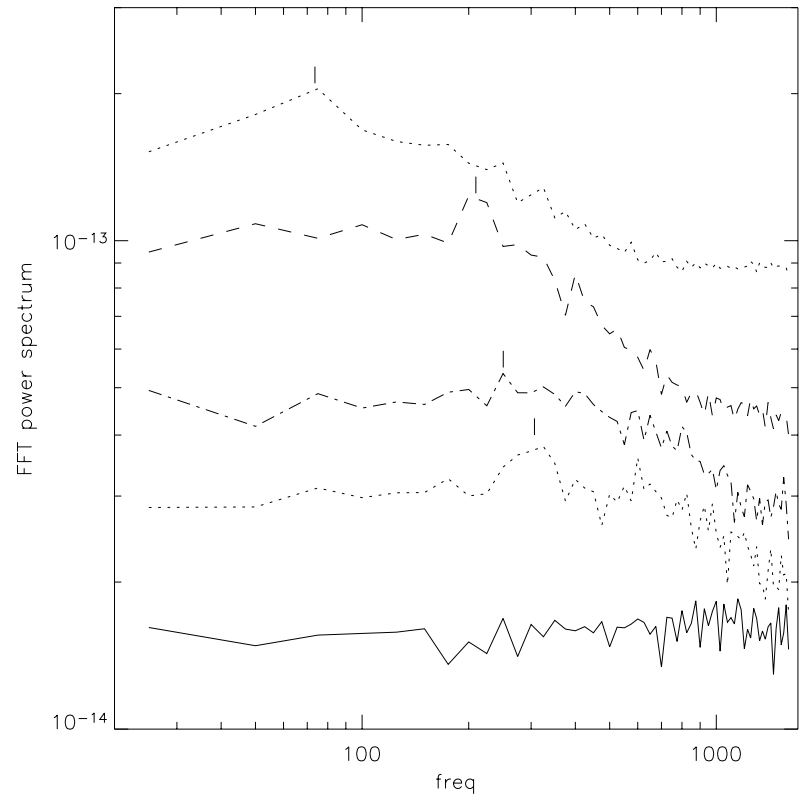

Fig. 4. Average power spectrum along $x$. Each curve corresponds to a different time of the simulation, i.e., from bottom to top: $t=0, t=$ $0.11, t=0.15, t=0.30, t=0.37$ orbit (to be compared with the first frames of Fig. 1). The small vertical bar indicates the approximate position of the maximum of each curve. The frequency is expressed as $\left(1 / \lambda \mathrm{AU}^{-1}\right)$. To simplify comparisons, an arbitrary vertical shift has been added to each curve.

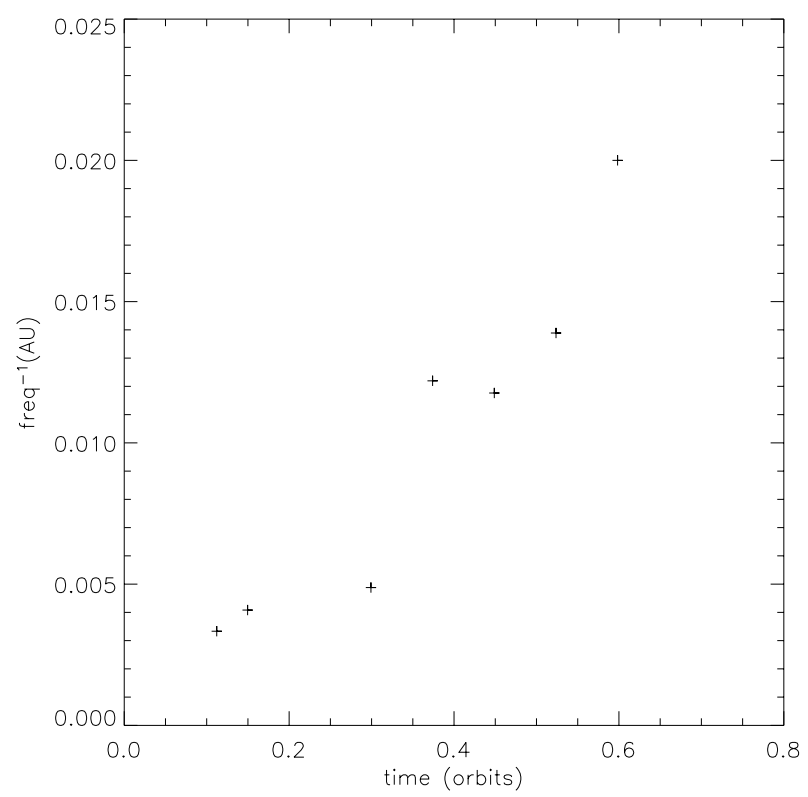

Fig. 5. Wavelength corresponding to the maximum of the power spectrum, as a function of time.

several orders of magnitudes higher than the timescale of the velocity dispersion increase observed here.

In other words, the development of the features observed on short timescales, corresponding to the transition from a dynamically cold, unstable disk, to a dynamically hot one, is practically independent of mutual collisions.

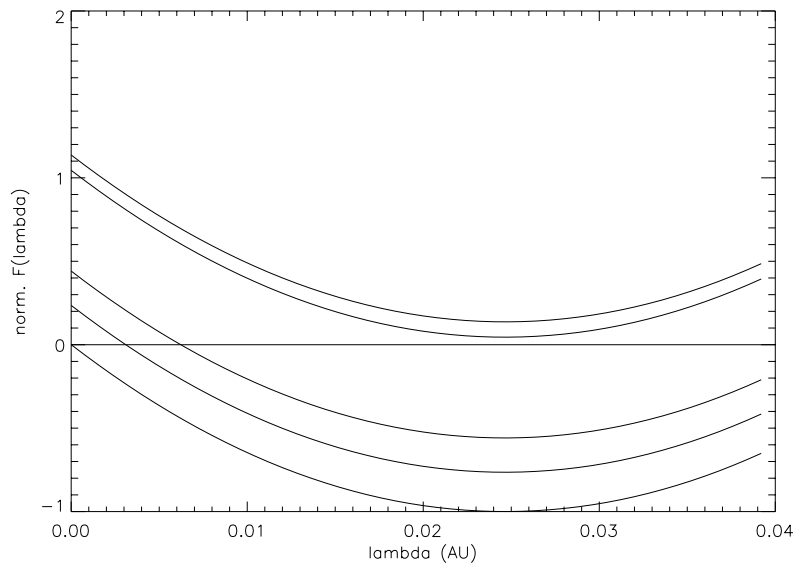

Fig. 6. The dispersion relation $F(\lambda)$ for the simulation presented in Fig. 1, at the corresponding times. The lower curve corresponds to the initial conditions.

\section{The instability in the presence of gas drag}

An efficient dissipation of kinetic energy can be obtained by introducing friction of the planetesimals from a gaseous nebula. In the following, we suppose that the gas flow, represented by the velocity $\boldsymbol{u}=\left(u_{x}, u_{y}, u_{z}\right)$, follows a purely laminar, Keplerian profile. In the local co-rotating system this can be written as:

$u_{x}=0 ; u_{y}=-\frac{3}{2} \Omega x ; u_{z}=0$.

The gas drag over the particle is as an additional acceleration having the form:

$\frac{\mathrm{d} \boldsymbol{v}}{\mathrm{d} t}=-\frac{1}{\tau_{\text {stop }}}(\boldsymbol{v}-\boldsymbol{u}(x, y, z))$,

where $\boldsymbol{u}$ is computed at the position of the particle $(x, y, z)$. For simplicity of computation, we assume that the stopping time is proportional to the size of the particle: $\tau_{\text {stop }}=R / \gamma, \gamma$ being a constant controlling the amount of gas friction. A given value of $\gamma$ will thus correspond to a specific stopping time for each particle size.

In the discussion following, however, we prefer to express gas drag through the Stokes parameter, defined as the ratio of the stopping time to the typical dynamical time of the fluid motion (the orbital period): $S t=\tau_{\text {stop }} \Omega / 2 \pi$. In our case, we will indicate the value of $S t$ for the initial particle size.

Depending on the value of $\tau_{\text {stop }}$, the particles will be more or less forced to follow the Keplerian shear (8). On the other hand, particles exactly moving as in Eq. (8) will not be influenced by any energy dissipation. As a result, no radial migration can be reproduced in this ideal case, and the only effect of the drag is to reduce velocity dispersions.

In the case of the minimum mass solar nebula, the gas drag would hardly have any effect on planetesimals with $R=10 \mathrm{~km}$ in the time span covered by the simulation presented here. Instead of imposing a realistic drag, we thus look for the smallest dissipation necessary to preserve some structures in the disk. Since the lifetime of the structures observed in the previous section is about $\sim 1$ orbit, we predict that a stopping time of the same order of magnitude should deeply affect their evolution. We thus test the evolution assuming $S t=1$. 

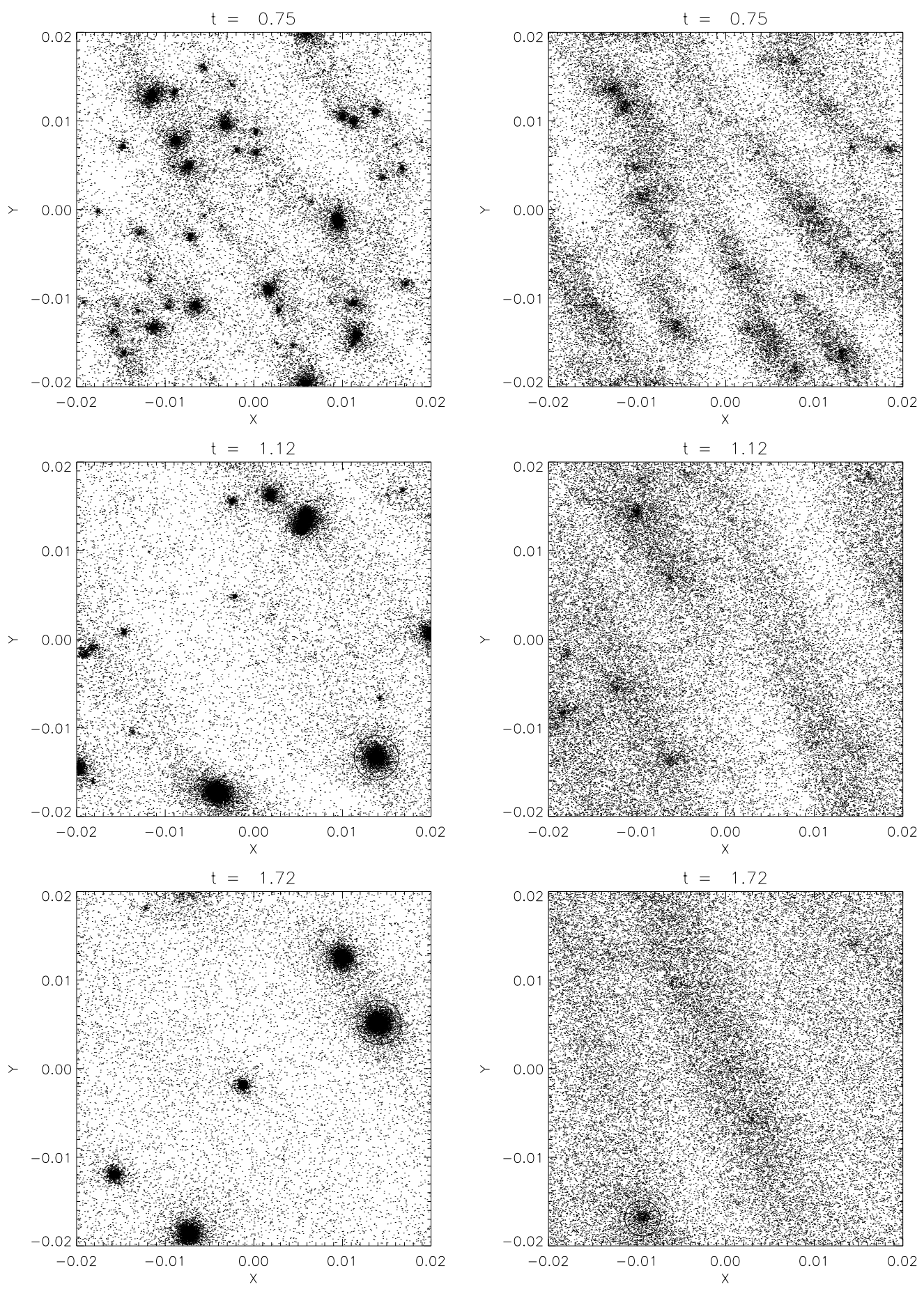

Fig. 7. Results from the CM simulation with $S t=1$ (left column) and $S t=10$ (right column), with merging particles. Large cluster merging is observed in the first case, while in the second one only a small spherical cluster survives at the end of the simulation. Circles in the left column snapshots represent the area used for computing detailed statistics, as detailed in the text.

In this case the effects of gas drag are readily apparent. Both in CM and EC simulations, the initial amplification of small wavelength fluctuations is followed by their hierarchical growth, as usual. The dissipation, however, prevents highdensity regions from dispersing, and the mutual interactions make clusters grow by merging. As can be seen qualitatively from the time evolution of the $(x, y)$ distribution of particles (Fig. 7, left column), the clusters evolve toward circular symmetry.
Except for the very beginning of the dynamical evolution, this kind of distribution is no longer well suited for characterization by the study of the Fourier transform, since a periodic density wave is not present. On the other hand, the two-point correlation function can be computed in order to give a statistical estimate of the degree of clustering. Formally, the two-point correlation function $\xi(r)$ is defined as the excess probability (relatively to a homogeneous distribution) of finding a point in a volume $\mathrm{d} r^{s}$ ( $s$ being the space topological dimension) at 
distance $r$ from a randomly chosen point. If $\delta P$ is the probability of finding a particle in the volume $\mathrm{d} r^{s}$, then:

$\delta P=\bar{n}[1+\xi(r)] \mathrm{d} r^{s}$,

in which $\bar{n}$ is the average number density of points in the distribution. Since we are mainly interested in the distribution on the $z=0$ plane, the computation of $\xi(r)$ was made in two dimensions $(s=2)$, by considering the positions of the particles projected on that plane.

Conventionally the length $\lambda_{h}$ for which $\xi\left(\lambda_{h}\right) \equiv 1$ is assumed to be the "characteristic length,", i.e., the scale of the largest typical clusters. From a practical point of view, we computed $\xi(r)$ by the method discussed in Rivolo (1986). Since boundary conditions are periodic, the procedure is exact in our case. The results obtained for four epochs are illustrated in Fig. 8. The strong growth of $\xi(r)$ at small distances is a clear sign of the increasing clustering. It can be noted that the probability of finding a particle close to another one (at a vanishing distance) grows to more than 40 times the average value. This value is averaged over the whole patch, thus including those particles that are not in the clusters. Nevertheless, since the fraction of clustered bodies is dominating the distribution, this can be considered to be a reliable estimate, at least at the end of the simulation. In the time interval considered, the characteristic length stabilizes around $\lambda_{h} \sim 2.5 \times 10^{-3} \mathrm{AU}$.

Taking this value for the average radius of the clusters, we can estimate their overall properties. At the latest time shown in (Fig. 7, left column), the cylinders of radius $\lambda_{h} \sim$ $2.5 \times 10^{-3}$ AU centered on the two larger clusters (in the upper right quadrant of the patch) include masses of $2.3 \times 10^{22} \mathrm{~g}$ and $3.6 \times 10^{22} \mathrm{~g}$. Both clusters include a member with a diameter larger than $100 \mathrm{~km}$ in their cores. If the entire mass was concentrated into single bodies of volume density $1 \mathrm{~g} / \mathrm{cm}^{3}$, they would have radii of 176 and $204 \mathrm{~km}$, respectively. The corresponding Hill radii would be $4.7 \times 10^{-3}$ AU and $5.4 \times 10^{-3}$ AU. At later times in the simulation the two clusters merge into a single one, containing about $40 \%$ of the total mass available in the patch. At the time reached by our longest simulations (i.e., $t \sim 4$ orbits) only two large clusters remain, whose average distance is larger than their Hill radius. However, we do not present here a detailed analysis of such end states, since to study the dynamics of such large clusters a much larger patch (of several Hill radii per side) would be necessary. We stress again here that the same qualitative behavior, on the same timescale, is observed when particle collisions are treated as bounces (EC case) or as merging events (CM).

In this last case it is interesting to check the properties of the evolving cumulative size distribution of particles. Figure 9 presents this distribution at two different times. Besides the global distribution, the size distribution restricted to a specific cluster is also computed. It can be seen that (especially at the most evolved stages) large members are present inside the clusters. We also computed the volume density on a cubic grid $N \times M \times L$ with $N=M=L=32$ and used it to study the relation between the size of a particle and the density of the cell in which it is contained. The existing correlation (Fig. 9, lower panel) indicates that large particles form and remain

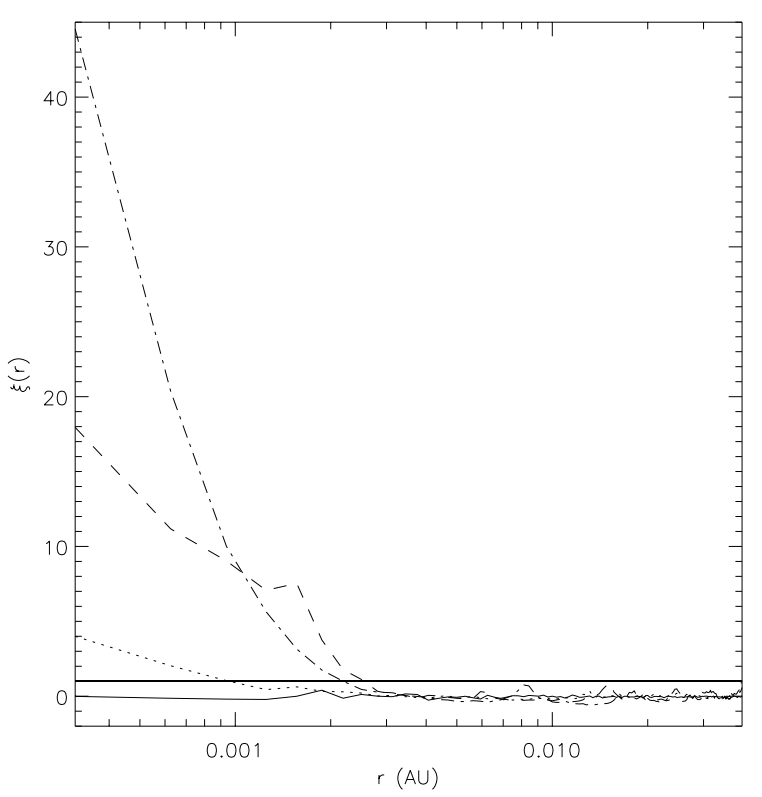

Fig. 8. The two-point correlation function at times $t=0, t=4.70, t=$ $7.28, t=10.81$ orbits. The line $\xi(r)=1$ has been added as a reference.

inside dense areas. We can thus state that particle growth is acting preferentially inside clusters.

Concerning some specific cluster properties, we note that:

a- The cluster shape is close to spherical. A plot of isodensity surfaces shows that a small flattening is indeed present at a distance of $\sim 2 \times 10^{-3}$ from the cluster center, but is negligible in its inner part.

b- The density profile in the central part of the cluster behaves like $\rho \sim r^{-2}$ (Fig. 10). This central region is about $10^{-3} \mathrm{AU}$ in size.

c- The orientation of the orbits around the cluster barycentre appear to be randomized. This can be seen by the fact that the velocities do not show any systematic orientation. We have analyzed the horizontal velocity $\boldsymbol{v}_{\mathrm{h}}$ after having subtracted the average Keplerian shear of each particle and having decomposed it into its radial and tangential components $\left(v_{r}, v_{t}\right)$ relative to the direction of the cluster barycentre. No trend can be identified by plotting $v_{r}$ or $v_{t}$ as a function of $r_{h}$ (distance from barycentre as projected on the disk equatorial plane). In addition, the direction of $v_{h}$ is uniformly distributed in the $[0,2 \pi]$ range. These statistics do not change if they are computed by considering all particles or only a subset of them, close to the disk average plane. The typical velocity dispersions close to the cluster center are very small, of the order of $\sigma_{v_{r}} \sim \sigma_{v_{t}} \sim 1.5 \mathrm{~m} \mathrm{~s}^{-1}$.

$\mathrm{d}-$ In the CM case, particle sizes are roughly sorted accordingly to their distance to the cluster center: large particles are close to the barycentre, while small bodies are scattered all around (Fig. 11). This behavior can be expected as the result of a sort of energy equipartition due to dynamical friction. We recall that, in flattened, stable disks, the effect of dynamical friction is to reduce the velocity dispersion of large bodies that are surrounded by a swarm of small particles. As a consequence, this swarm is "heated" and its velocity dispersion increases. From the point of view 

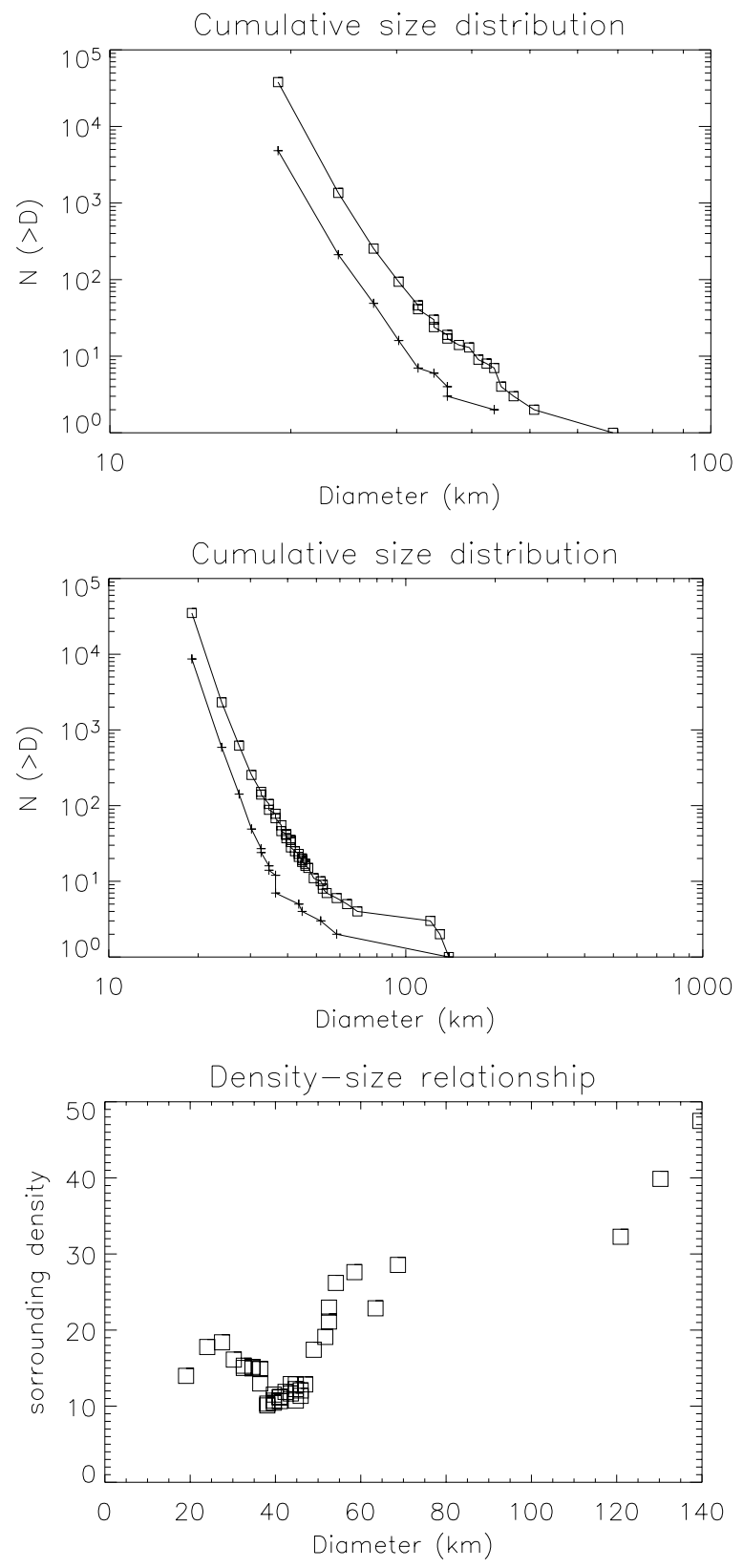

Fig. 9. Some statistics concerning the size distribution of the simulation in Fig. 7 (left column). The upper and middle panels show the size distribution at $t=7.05$ and $t=7.15$ orbits, respectively. The upper curve is the distribution obtained when including all particles. The lower curve is the distribution referred to the domain inside the corresponding circle in Fig. 7 (lower left panel). The plot of particle size vs. local density (lower panel), shows the tendency of particles to grow faster in overdense regions, and is referred to time $t=10.81$ orbits.

of the vertical distribution relative to the average plane, this translates into the large particles being less dispersed than the small ones. Inside the clusters studied here, the mechanism is the same, except that the system has a spherical symmetry.

e- According to these dynamics, runaway growth is present, with the largest body detaching from the distribution and growing faster.

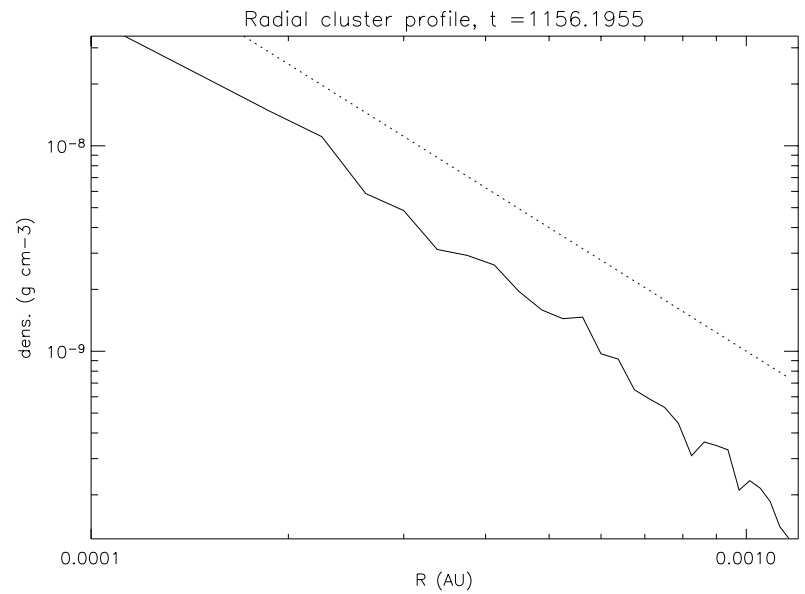

Fig. 10. The average radial density profile (solid line) in the most dense part of the cluster circled in Fig. 7 (middle panel, left column). The dotted line represents the power law $R^{-2}$.

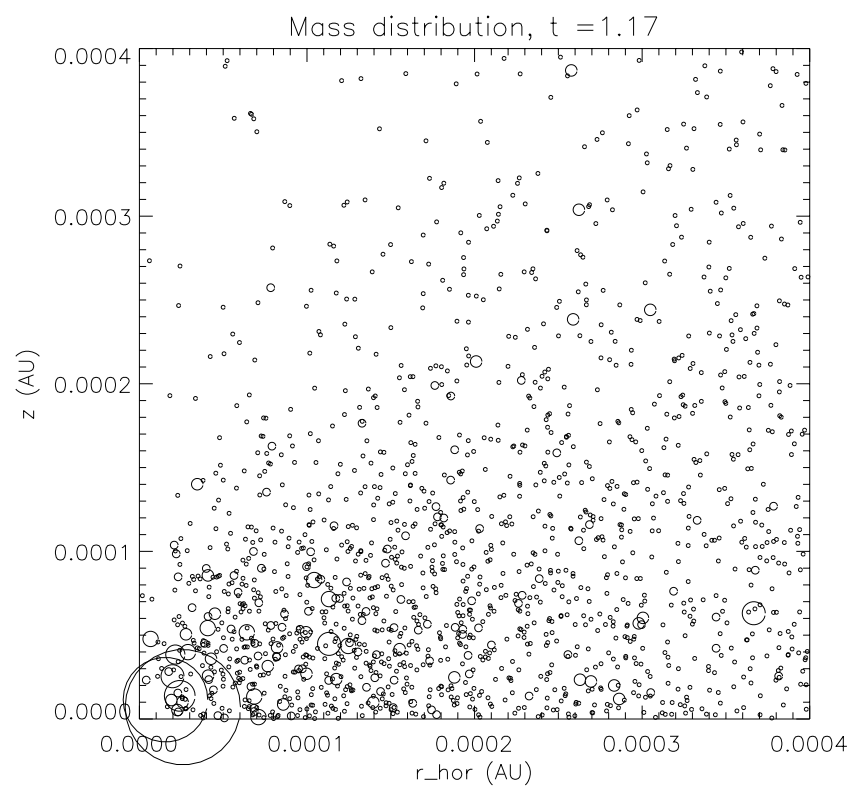

Fig. 11. The particle distribution in the most dense part of the cluster circled in Fig. 7 (middle panel, left column). Each circle is centered on the position of a particle in the $(r, z)$ plane; the radius is proportional to the mass of the particle in order to emphasize the effect of size differences. As can be seen, the center of the cluster is populated by large bodies.

It must be noted that both the radial profile and the randomization of velocities are in agreement with that of a selfgravitating, completely virialized cluster. The $\rho \sim r^{-2}$ density distribution was suggested for virialized halos with isothermal profiles (Gunn \& Gott 1972). Indirectly, the virialization is confirmed by the fact that, given the order of magnitude of the cluster size, the typical cluster crossing time for a particle is $t_{\text {cross }} \sim 2.5 \times 10^{-3}$ orbits, i.e., several times less than the cluster lifetime observed in our simulations. The corresponding two-body relaxation time for globular clusters composed of $N_{\text {clus }}$ bodies is usually computed as $t_{\text {rel }} \sim 0.1 \frac{N_{\text {clus }}}{\ln N_{\text {clus }}} t_{\text {cross }}$. In our most populated clusters, $N_{\text {clus }} \sim 10^{4}$, yielding $t_{\text {rel }} \sim 0.64$ orbits, i.e., less than the duration of our simulations. 
Furthermore, it must be noted that the clusters interact and merge on a timescale of a few orbits. During this interval, the dynamics is not affected by collisions, being comparable both in the $\mathrm{CM}$ and the EC scenario.

We also verified that $S t \sim 1$ corresponds to the gas drag that maximally preserves the structures, by running some simulations with higher and lower values of $\gamma$, in power-of-ten steps. In general, we observe that with a very strong drag $\left(S t=10^{-3}\right)$, as expected, all velocity dispersions are rapidly damped. The vertical distribution becomes very thin and close encounters are favored by the nearly two-dimensional distribution. Due to small relative velocities and increasingly strong gravitational focusing, mutual collisions are frequent. If merging is allowed, the masses of the particles grow fast. This particular simulation ends at $t=3800$ with $N$ decreased to $N=980$, and without the formation of any structures.

Only at $S t=0.1$ do clusters of particles begin to emerge from the distribution. The velocity dispersion increases with time, and accretion is still very efficient. However, a large fraction of particles remains outside the clusters. It can be seen that a strong correlation exists between the size of the particles and the surrounding volume density of solids (Fig. 12).

On the other hand, for larger stopping times $(S t=10)$ it is interesting to note that the transient formation of several clusters is present. However, instead of merging, they rapidly disappear due to gravitational stirring. In the case of our simulation, we observe one remaining condensation (containing only about $2.3 \%$ of the total mass available), indicating that cluster survival is at least marginally allowed (Fig. 7, right column).

Even longer stopping times will yield a drag insufficient to promote cluster formation.

We also tested the formation of clusters starting with completely different initial conditions, i.e. with the "hot disk" shown in Fig. 1 (bottom right panel), and allowing the gas to operate the "cooling" necessary to drive the system to the unstable regime. Some differences in the details of the evolution exist. In particular, due to the higher velocity dispersion, the instability does not take place at small scales, but directly at a scale close to $\lambda_{\mathrm{c}}$. Details of this process will be further investigated; here we just want to stress that, even in this "inverse" case, clusters form and have the same properties as presented in this work.

\section{Conclusions}

The system explored, when no dissipation is acting, evolves spontaneously toward a stable configuration in which velocity dispersions are too high to allow any gravitational instability. This is clearly shown in the simulation presented in Fig. 1. As already discussed, mutual collisions are not able to damp velocity dispersions efficiently, and play a secondary role over the observed dynamical evolution. However, this can be regarded as a consequence of the particular regime that has been chosen. In fact, closer to the sun and/or using much smaller particles, the dynamics could be collision-dominated (Furuya et al. 2002).

Our simulations underline the role of both the non-linear evolution of the collapsing layer and of its granularity. Thanks
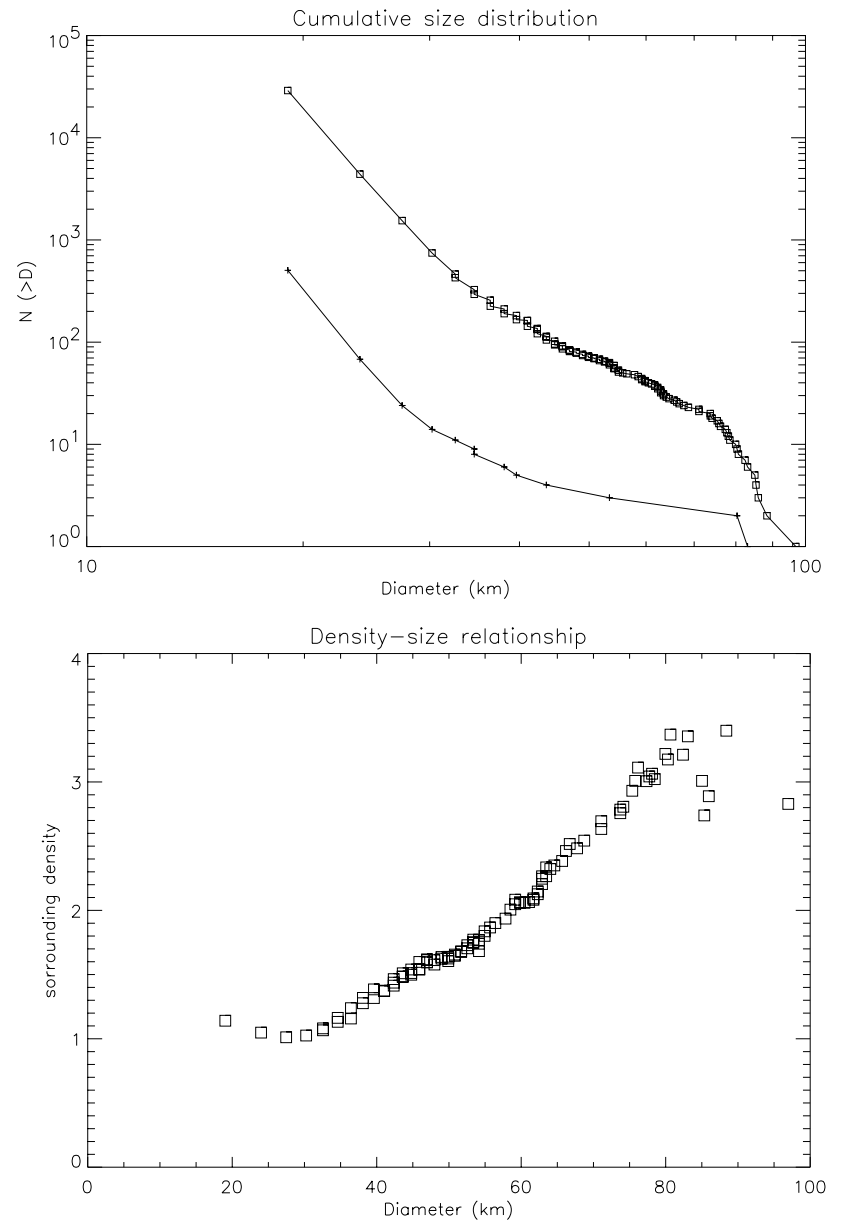

Fig. 12. Some results from the CM simulation with $S t=0.1$. The upper panel shows the final cumulative size distribution: the upper curve represents the distribution computed by taking into account all the particles. The lower curve takes into account only particles belonging to the larger cluster. The bottom panel shows that a correlation between the size of the particle and the surrounding volume density is present.

to the hierarchical growth of structures, the particle-particle interaction is gradually substituted by the cluster-cluster one at larger scales. At the end of the simulations that include gas drag, large clusters behave as super-particles dominating the dynamics. A first set of tests, made by isolating a single cluster from the surrounding particles and computing its evolution on long times, seems to show that the clusters are intrinsically "stable" structures. Gas friction also guarantees that they can shrink and collapse to form large bodies, although on time scales much larger than those explored here.

Of course, our approach is also affected by some limitations. A strong limit of our study concerns the use of a layer of equally sized particles representing the solid component. It has been suggested that a full range of particle sizes, exhibiting different couplings to the nebular gas, could deeply affect the instability of the disk (Weidenschilling 1995; Ward 2000). This point will be investigated in a coming paper.

Furthermore, in order to study the dynamics of larger bodies in the presence of dissipation, we introduced a gas drag law with intensity not physically related to particle sizes. In other words, the resulting stopping time was much smaller than any 
realistic value that could correspond to $\mathrm{km}$-sized planetesimals in the environment of a classical protoplanetary disk.

On the other hand, it must be noted that the formation of self-gravitating, virialized clusters is not specific to this choice. In fact, the dynamics observed is essentially collisionless, being unaltered by the presence of merging or perfectly bouncing particles. Thus, as commonly done in cosmology, we can assume that our discrete distribution of large bodies just represents a single possible sampling of the density field. Probably, its dynamics would be qualitatively equivalent if the field was sampled by much smaller bodies, as long as long-range interactions are possible (i.e., the Hill radius is much larger than the physical radius) and the stopping time is comparable to the orbital period $(S t \sim 1)$. In a "realistic" minimum-mass nebula, this could be true for particle sizes of the order of $R \sim 1 \mathrm{~m}$, sufficiently far from the sun (in order to satisfy the Hill radius constraint), provided the radial velocity dispersion can be neglected - which would not always be the case. Under these conditions, the mechanism illustrated here could thus dominate the dynamics of an unstable layer of solids. Further investigations are under way following this direction, and will be the subject of a future publication.

Another major drawback in the direct application of this scenario to the growth of planetesimals is related to the fact that the action of particles over gas is neglected. In fact, it is clear that, when solids are strongly concentrated inside clusters, they could deeply affect the motion of the surrounding gas. This feedback was already invoked as a cause of stabilization of the midplane dust layer, through the stirring operated by the turbulence. In that scenario, the main cause of turbulence is the shear due to the systematic velocity difference between dust particles and gas. In our case, since cluster member velocities are randomized, no net systematic motion would be induced in the average gas flow. However some kind of smallscale turbulence, with the associated diffusivity, could oppose the collapse.

In conclusion, we want to underline the observation, in our numerical simulations, of the extremely rich dynamics hidden behind gravitational instability in a Keplerian disk. Even though the application to the study of planetesimal growth requires further effort, the phenomena exposed here may always be present when collective effects, rather than collisional effects, dominate the dynamics.

Acknowledgements. This study was supported in part by the Programme Nationale de Planétologie. D. Richardson acknowledges support of the NASA Origins of Solar Systems contract \#NAG-511722. S. J. Weidenschilling was supported by the NASA Planetary Geology and Geophysics Program, Grant \#NAG5-13156. We thank the IDRIS computing center facility for the use of the SP3 and SP4 parallel computers. SIVAM and SIVAM II computing systems at the OCA were also used for this work. We wish also to acknowledge useful discussions with P.-H. Chavanis and G. Murante, and the work of an anonymous referee.

\section{References}

Barge, P., \& Sommeria, J. 1995, A\&A, 295(1), L1

Edgeworth, K. E. 1949, MNRAS, 109, 600

Furuya, I., Daisaka, H., \& Nakagawa, Y. 2002, in Proc. IAU 8th AsianPacific Regional Meeting, Tokyo, 2, 21

Goldreich, P., \& Ward, W. R. 1973, ApJ, 183, 1051

Goodman, J., \& Pindor, B. 2000, Icarus, 148, 537

Gunn, J. E., \& Gott, J. R. 1972, ApJ, 176, 1

Richardson, D. C. 1994, MNRAS, 269(2), 493

Richardson, D. C., Quinn, T., Stadel, J., \& Lake, G. 2000, Icarus, 143, 45

Rivolo, A. R. 1986, AJ, 301, 70

Safronov, V. S. 1969, Evolution of the protoplanetary cloud and formation of the Earth and the planets (Moskow: Nauka)

Tanga, P., Babiano, A., Dubrulle, B., \& Provenzale, A. 1996, Icarus, 121,158

Tanga, P., Michel, P., \& Richardson, D. C. 2002, A\&A, 315, 613

Ward, W. R. 1976, in Frontiers of Astrophysics, ed. E. H. Avrett (Cambridge: Harvard Univ. Press)

Ward, W. R. 2000, in Origin of the Earth and Moon, ed. R. M Canup, $\&$ K. Righter (Tucson: Univ. of Arizona Press)

Wisdom, J., \& Tremaine, S. 1988, AJ, 95, 925

Weidenschilling, S. J. 1980, Icarus, 44, 172

Weidenschilling, S. J. 1995, Icarus, 116, 433

Weidenschilling, S. J. 1997, Icarus, 127, 290

Weidenschilling, S. J. 2003, Icarus, 165, 438

Youdin, A. N., \& Shu, F. H. 2002, ApJ, 580, 494 